\title{
Is diffusion-weighted magnetic resonance imaging superior to positron emission tomography with fludeoxyglucose $F 18$ in imaging non-small cell lung cancer?
}

\author{
Yasuomi Ohba, MD, ${ }^{\text {a }}$ Hiroaki Nomori, MD, PhD,,${ }^{\text {ad }}$ Takeshi Mori, MD, PhD, ${ }^{\text {a }}$ Koei Ikeda, MD, PhD, ${ }^{\text {a }}$ \\ Hidekatsu Shibata, MD, ${ }^{\mathrm{a}}$ Hironori Kobayashi, MD, PhD, ${ }^{\mathrm{a}}$ Shinya Shiraishi, MD, $\mathrm{PhD},{ }^{\mathrm{b}}$ and \\ Kazuhiro Katahira, $\mathrm{MD}, \mathrm{PhD}^{\mathrm{c}}$
}

Objective: This retrospective analysis examined whether diffusion-weighted magnetic resonance imaging might be as useful as positron emission tomography with fludeoxyglucose $\mathrm{F} 18$ for (1) discriminating between non-small cell lung cancer and benign pulmonary nodules and (2) predicting aggressiveness of non-small cell lung cancer.

\begin{abstract}
Methods: Diffusion-weighted magnetic resonance imaging and positron emission tomography were performed before surgery in 110 patients with 124 pulmonary nodules smaller than $3 \mathrm{~cm}$, including 96 non-small cell lung cancers and 28 benign nodules. Diffusion of water molecules in magnetic resonance imaging was measured by minimum value of apparent diffusion coefficient. The criterion standard was the result of histologic diagnosis or follow-up examination. Sensitivity and specificity for differentiating between cancers and benign nodules were compared between diffusion-weighted imaging and positron emission tomography. Apparent diffusion coefficient in diffusion-weighted imaging and fludeoxyglucose F 18 uptake in positron emission tomography were examined with respect to pathologic tumor stage; lymphatic, vascular and pleural involvements; and histologic differentiation.
\end{abstract}

\begin{abstract}
Results: There were no significant differences between diffusion-weighted magnetic resonance imaging and positron emission tomography in sensitivity or specificity for non-small cell lung cancer. Whereas positron emission tomography showed significant differences in fludeoxyglucose F 18 uptake between pathologic stages IA versus IB or more advanced stages; between tumors with and without lymphatic, vascular, or pleural involvement; and between well-differentiated and moderately or poorly differentiated adenocarcinomas $(P<.01-0.001)$, no significant differences in apparent diffusion coefficient values in were observed.
\end{abstract}

Conclusion: Diffusion-weighted magnetic resonance imaging is equivalent to positron emission tomography in distinguishing non-small cell lung cancer from benign pulmonary nodules but is not as useful for predicting aggressiveness of non-small cell lung cancer.

Recent advances in magnetic resonance (MR) gradient technology have led to the introduction of diffusion-weighted MR imaging (DWI), which provides excellent tissue contrast through detection of differences in the diffusion of water molecules among tissues and is entirely different from ordinary T1- and T2-weighted MR images. Because the diffusion of water molecules is disturbed by intracellular organelles and macromolecules, any architectural changes in the

\footnotetext{
From the Departments of Thoracic Surgery a and Diagnostic Radiology, ${ }^{\mathrm{b}}$ Graduate School of Medical Sciences, Kumamoto University, Kumamoto, Japan; and the Department of Radiology, Kumamoto Chuo Hospital, ${ }^{\mathrm{c}}$ Kumamoto, Japan; and the Division of General Thoracic Surgery, Department of Surgery, School of Medicine, Keio University, ${ }^{\mathrm{d}}$ Tokyo, Japan.

Received for publication April 27, 2008; revisions received Oct 11, 2008; accepted for publication Dec 27, 2008; available ahead of print March 31, 2009.

Address for reprints: Hiroaki Nomori, MD, PhD, Department of Thoracic Surgery, Graduate School of Medical Sciences, Kumamoto University, 1-1-1 Honjo, Kumamoto 860-8556, Japan (E-mail: hnomori@qk9.so-net.ne.jp).

J Thorac Cardiovasc Surg 2009;138:439-45

0022-5223/\$36.00

Copyright (c) 2009 by The American Association for Thoracic Surgery

doi:10.1016/j.jtcvs.2008.12.026
}

proportion of extracellular to intracellular water molecules alters the signal intensity on DWI, the apparent diffusion coefficient (ADC). ${ }^{1,2}$ Because malignant tumors are characterized by increased cellularity, larger nuclei with more abundant macromolecular proteins, larger nuclear/cytoplasmic ratio, and less extracellular space relative to normal tissues, the diffusion of water molecules in malignant tumors is restricted, resulting in decreased ADC values and allowing imaging of malignant tumors by DWI. ${ }^{3,4}$ To date, the utility of DWI has been reported for the imaging of brain tumors, ${ }^{5}$ breast tumors, ${ }^{6}$ musculoskeletal tumors, ${ }^{7}$ prostate cancer, ${ }^{8}$ rectal cancer, ${ }^{9}$ and lung cancer. ${ }^{10}$

Positron emission tomography (PET) with fludeoxyglucose F 18 (FDG) has been found to be extremely useful for discriminating between malignant and benign pulmonary nodules. Although several authors have compared images between DWI and PET in a small number of tumors, ${ }^{11,12}$ there have been few reported comparisons of the sensitivity and specificity of DWI and FDG-PET for diagnosing malignancy. Recently, we showed DWI to have similar sensitivity 


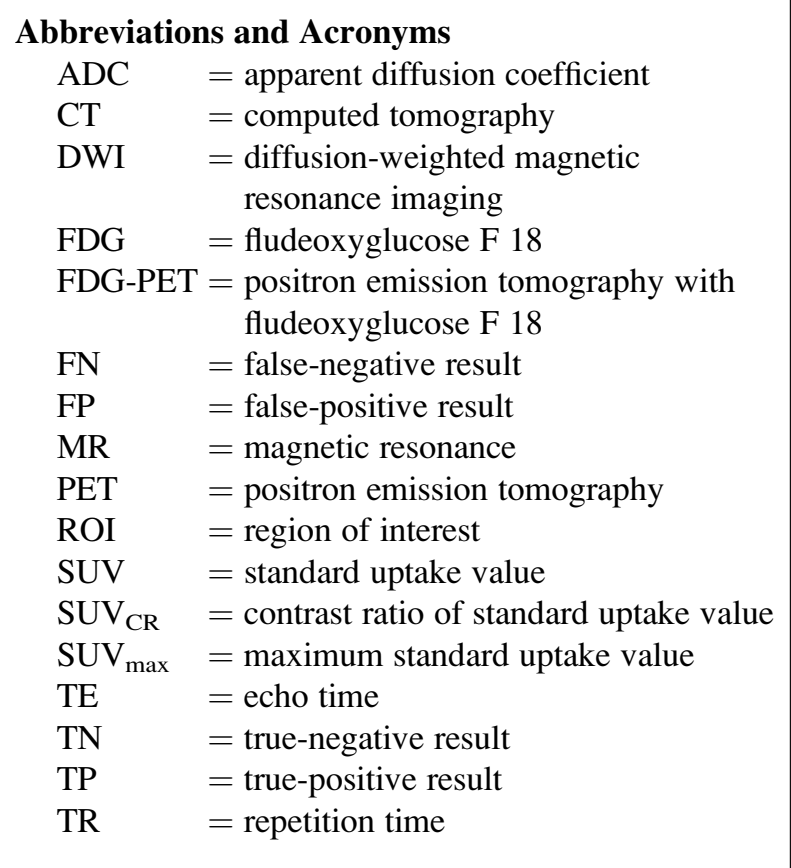

and a slightly higher specificity than FDG-PET for distinguishing between malignant and benign pulmonary nodules. ${ }^{13}$ Ohno and colleagues ${ }^{14}$ reported that whole-body MR imaging with DWI had similar accuracy to FDG-PET for M-staging in patients with non-small cell lung cancer (NSCLC).${ }^{14}$ FDG-PET has also been reported to aid in assessment of tumor aggressiveness, however, including tumor stage and intratumoral invasiveness, and thus prognosis. ${ }^{15-18}$ In assessing the relative merits of DWI and FDGPET for imaging of NSCLC, the utilities of the examinations for both diagnosis and evaluation of tumor aggressiveness should be compared.

In this study, we conducted a retrospective analysis of the subject data with the following objectives: (1) to examine the utility of DWI in the differential diagnosis of pulmonary nodules by comparing the sensitivities and specificities of DWI and FDG-PET for discriminating between NSCLC and benign pulmonary nodules, and (2) to compare the utility of DWI and PET in predicting the aggressiveness of NSCLC. Although our previous study included both primary and metastatic lung cancers ${ }^{13}$ metastatic lung cancers were excluded in this study to compare the utility of the two procedures in evaluating the aggressiveness of NSCLC. In addition, although our previous study included tumors larger than $3 \mathrm{~cm}$, this study was targeted at NSCLCs smaller than 3 $\mathrm{cm}$ to compare the utility in assessing tumor aggressiveness between DWI and FDG-PET, because FDG uptake is dependent on the tumor size. ${ }^{19}$

\section{MATERIALS AND METHODS Eligibility}

The examination of both DWI and FDG-PET in patients with lung cancer was approved by the Ethical Committee of Kumamoto University Hospital in January 2006. Informed consent was obtained from all patients after they and their surgeons received explanations of the costs and benefits of the examinations.

\section{Patients}

Between February 2006 and December 2007, a total of 144 patients with 182 pulmonary lesions underwent both FDG-PET and DWI, which were done within 2 weeks of each other. Of these lesions, 22 lesions larger than $3 \mathrm{~cm}$ and 11 metastatic lung cancers were excluded from this study. Twenty-five lesions with the appearance of pure ground-glass opacity were also excluded, because all these nodules yielded negative results on both DWI and PET. After these exclusions, 110 patients with a total of 124 nodules were entered in the study (Table 1). There were 56 men and 54 women, with a mean age of $68 \pm 9$ years (range 36-82 years). Of the 124 nodules, 106 were diagnosed histologically after surgical resection, whereas 18 were diagnosed as old inflammation because their sizes were found on review of retrospective chest radiography or computed tomography (CT) to have been unchanged for more than 2 years. Mean follow-up duration for these 18 nodules, of which 6 were checked by radiography and the other 12 by CT, was $41 \pm 23$ months (range: $24-97$ months). Mean size of these 18 nodules was $1.6 \pm 0.6 \mathrm{~cm}$ (range $1.0-3.0 \mathrm{~cm}$ ). None of the 124 lesions had apparent cavitations and calcifications on CT. Long-axis diameters of pulmonary nodules were considered to represent sizes of the lesions.

\section{PET and CT Scanning}

Patients were instructed to fast for at least 5 hours before intravenous administration of FDG. The dose of FDG administered was $100 \mu \mathrm{Ci} / \mathrm{kg}$ (3.7 $\mathrm{MBq} / \mathrm{kg}$ ). PET imaging was performed approximately 60 minutes after intravenous administration of FDG with an integrated PET and CT device (Discovery ST; GE Medical Systems, Milwaukee, Wis) that consisted of a PET scanner (Advance Nx; GE Medical Systems) and an 8-section CT scanner (Light Speed Plus; GE Medical Systems). All images were acquired under shallow-breathing conditions.

The acquisition time for PET in 3-dimensional mode was 3 minutes per table position. CT data were resized from a $512 \times 512$ matrix to a $128 \times 128$ matrix to match the PET data to allow image fusion, and a CT transmission map was generated. PET image data were reconstructed iteratively with the ordered subsets expectation maximization algorithm with segmented attenuation correction ( 4 iterations, 28 subsets) and the CT data. The 3.75 -mm thick transaxial CT images were reconstructed at 3.27- $\mathrm{mm}$ intervals (transaxial) for fusion with the transaxial PET images. The PET, CT, and fused images were available for review in the axial, coronal, and sagittal planes by means of software (Xeleris; GE Medical Systems) on a computer workstation.

\section{Diffusion-Weighted Imaging}

All MR images were obtained with a 1.5-T superconducting system (Gyroscan Intera Achieva Nova Dual; Philips Nederland BV Medical Systems, Eindhoven, The Netherlands). Conventional MR and DWI images were acquired during the same procedure. The conventional MR images consisted of a coronal T1-weighted sequence (repetition time [TR] 234 $\mathrm{ms}$, echo time [TE] $4 \mathrm{~ms}, 1$ excitation) and coronal and axial single-shot spin echo T2-weighted (TR $800 \mathrm{~ms}$, TE $90 \mathrm{~ms}, 1$ excitation) and coronal and axial short tau inversion recovery (TR $4600 \mathrm{~ms}$, TE $90 \mathrm{~ms}$, inversion time $160 \mathrm{~ms}$ ) sequences. The T1-weighted, T2-weighted, and short tau inversion recovery sequences were acquired at a section thickness of $6 \mathrm{~mm}$ with a $1-\mathrm{mm}$ intersection gap, a $128 \times 128$ matrix, and a 40 - to 45 -cm field of view. All images were acquired under shallow-breathing conditions.

DWI was performed in the transverse plane with a spin-echo, echo-planar imaging sequence with the following parameters: TR $5900 \mathrm{~ms}$, TE 60 
TABLE 1. Characteristics of non-small cell lung cancer and benign nodules

\begin{tabular}{|c|c|}
\hline \multicolumn{2}{|l|}{ Non-small cell lung cancer $(n=96)$} \\
\hline \multicolumn{2}{|l|}{ Size $(\mathrm{cm})$} \\
\hline Mean \pm SD & $1.6 \pm 0.7$ \\
\hline Range & $1.0-3.0$ \\
\hline \multicolumn{2}{|l|}{ Histologic type (No.) } \\
\hline Adenocarcinoma & 76 \\
\hline Well differentiated & 57 \\
\hline Moderately differentiated & 14 \\
\hline Poorly differentiated & 5 \\
\hline Squamous cell carcinoma & 17 \\
\hline Adenosquamous carcinoma & 3 \\
\hline \multicolumn{2}{|l|}{ Pathologic stage (No.) } \\
\hline IA & 75 \\
\hline IB-IV & 21 \\
\hline T1N1M0 & 5 \\
\hline T3N0M0 & 2 \\
\hline T3N1M0 & 3 \\
\hline $\mathrm{T} 1 \mathrm{~N} 2 \mathrm{M} 0$ & 4 \\
\hline T3N2M0 & 3 \\
\hline T4N2M0 & 3 \\
\hline T1N1M1 & 1 \\
\hline \multicolumn{2}{|l|}{ Benign nodules $(\mathrm{n}=28)$} \\
\hline \multicolumn{2}{|l|}{ Size $(\mathrm{cm})$} \\
\hline Mean \pm SD & $1.5 \pm 0.7$ \\
\hline Range & $1.0-3.0$ \\
\hline \multicolumn{2}{|l|}{ Type } \\
\hline Acute inflammation & 4 \\
\hline Chronic inflammation & 20 \\
\hline Hamartoma & 2 \\
\hline Others & 2 \\
\hline
\end{tabular}

$\mathrm{ms}$, flip angle $90^{\circ}$, diffusion gradient encoding in 3 orthogonal directions, $\mathrm{b}=1000 \mathrm{~s} / \mathrm{mm}^{2}$, field of view $400 \mathrm{~mm}$, matrix size $112 \times 100$, section thickness $6 \mathrm{~mm}$, section gap $1 \mathrm{~mm}$, and number of signals acquired 6 .

\section{PET Data Analysis}

One radiologist (S.S.) with 11 years of radioisotope scintigraphy and PET experience who was unaware of the patients' clinical data evaluated the PET and CT data. After image reconstruction, a 2-dimensional circular region of interest (ROI) was drawn in a slice after visual detection of the highest count on the fused CT image. For the lesions with negative or faintly positive PET findings, the ROI was drawn on the fusion image with the corresponding CT. From these ROIs, the standard uptake value (SUV) maximum $\left(\mathrm{SUV}_{\max }\right)$ was calculated. The contrast ratio of SUV $\left(\mathrm{SUV}_{\mathrm{CR}}\right)$ was then calculated as described previously. ${ }^{20,21}$ Briefly, the values of SUV $\max$ in the tumor ROI (T) and at the equivalent point in the contralateral normal lung $(\mathrm{N})$ were measured. The $\mathrm{SUV}_{\mathrm{CR}}$ was calculated as $(\mathrm{T}-\mathrm{N}) /(\mathrm{T}+\mathrm{N})$ in each lesion as an index of FDG uptake.

\section{DWI Data Analysis}

One radiologist (K.K.) with 17 years of MRI experience who was unaware of the patients' clinical data evaluated the MRI image. DWI data were evaluated semiquantitatively with ADC. The ADC was calculated as follows: $\mathrm{ADC}=-\left[\ln \left(\mathrm{S} / \mathrm{S}_{0}\right)\right] / \mathrm{b}$, where $S$ is the signal intensity of the ROI obtained through 3 orthogonally oriented DWIs or diffusion trace images, and $b$ is a gradient $b$ factor with a value of $1000 \mathrm{~s} / \mathrm{mm}^{2}$. After image reconstruction, a 2-dimensional square ROI was drawn in a slice where the lesion was detected visually with reference to the T2-weighted image on CT. From the
ROI, the minimum ADC value was calculated. The mean ADC was not used, because the value within the entire tumor might not characterize the tumor as a result of heterogeneity. ${ }^{22}$

\section{Determining Cutoffs for $\mathrm{ADC}$ and $\mathrm{SUV}_{\mathrm{CR}}$}

A receiver operating characteristic curve was constructed according to

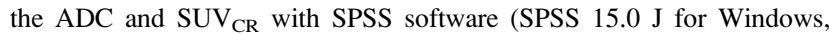
SPSS Inc, Chicago, Ill), and the cutoffs were determined for discrimination between benign and malignant lesions. Nodules with less than the ADC cutoff were defined as positive on DWI. Nodules with more than the $\mathrm{SUV}_{\mathrm{CR}}$ cutoff were defined as positive on PET and CT.

\section{Pathologic Analysis}

Hematoxylin and eosin and elastica van Gieson staining were performed on all sections to investigate lymphatic, vascular, and pleural involvements. Histologic differentiation in adenocarcinoma was classified into well-differentiated, moderately differentiated, and poorly differentiated grades. Pleural involvement was classified as $\mathrm{p} 0, \mathrm{p} 1, \mathrm{p} 2$, and $\mathrm{p} 3$; that is, a p0 tumor did not extend beyond the pleural elastic layer, a p1 tumor invaded the visceral pleural elastic layer but did not reach the pleural surface, a p2 tumor included tumor exposure on the pleural surface, and a $\mathrm{p} 3$ tumor invaded the parietal pleura or the chest wall. Pathologic tumor stages were based on the TNM classification of the International Union Against Cancer $^{23}$ : p2 tumors were classified as T2, p3 tumors were classified as T3, and tumors with intrapulmonary metastases within the same lobe were classified as $\mathrm{T} 4$.

\section{Analysis of Tumor Aggressiveness}

The aggressiveness of NSCLC was evaluated according to pathologic tumor stage, tumor invasiveness (as determined by lymphatic, vascular, or pleural involvement), and histologic differentiation of adenocarcinoma, because these factors are known to be related to prognosis. ${ }^{24,25}$ To evaluate the utility of DWI and PET in predicting tumor aggressiveness, the ADC and the $\mathrm{SUV}_{\mathrm{CR}}$ were compared between pathologic stage IA versus stage IB or more advanced stages; between tumors with and without lymphatic, vascular, or pleural involvement; and between well-differentiated and moderately or poorly differentiated adenocarcinomas.

\section{Statistical Analysis}

The differences in sensitivity and specificity between DWI and PET were analyzed with the McNemar test. True-positive (TP), true-negative (TN), false-positive (FP), and false-negative (FN) results of PET with CT and DWI images for detecting pulmonary malignancies were compared with the results of pathologic diagnosis or follow-up examination. Sensitivity was calculated as $\mathrm{TP} /(\mathrm{TP}+\mathrm{FN})$, specificity as $\mathrm{TN} /(\mathrm{TN}+\mathrm{FP})$, and accuracy as $(\mathrm{TP}+\mathrm{TN}) /$ total. Positive and negative predictive values were calculated as $\mathrm{TP} /(\mathrm{TP}+\mathrm{FP})$ and $\mathrm{TN} /(\mathrm{TN}+\mathrm{FN})$, respectively, and differences between DWI and PET were analyzed with the Student $t$ test. The Student $t$ test was also used to compare size distribution between malignant and benign nodules, $\mathrm{ADC}$ and $\mathrm{SUV}_{\mathrm{CR}}$ at pathological stages IA versus stage IB or more advanced stages, and $\mathrm{ADC}$ and $\mathrm{SUV}_{\mathrm{CR}}$ between tumors that were or were not invasive. Statistical analysis was performed with SPSS software. All values in the text and tables are given as mean $\pm \mathrm{SD}$.

\section{RESULTS}

Histologic type of NSCLC was adenocarcinoma in 76 lesions, squamous cell carcinoma in 17 , and adenosquamous carcinoma in 3. Adenocarcinoma was well differentiated in 57 lesions, moderately differentiated in 14 , and poorly differentiated in 5. Pathologic stage was IA in 75 lesions and IB or a more advanced stage in 21 (Table 1). Benign nodules showed acute inflammation in 4 lesions, chronic 

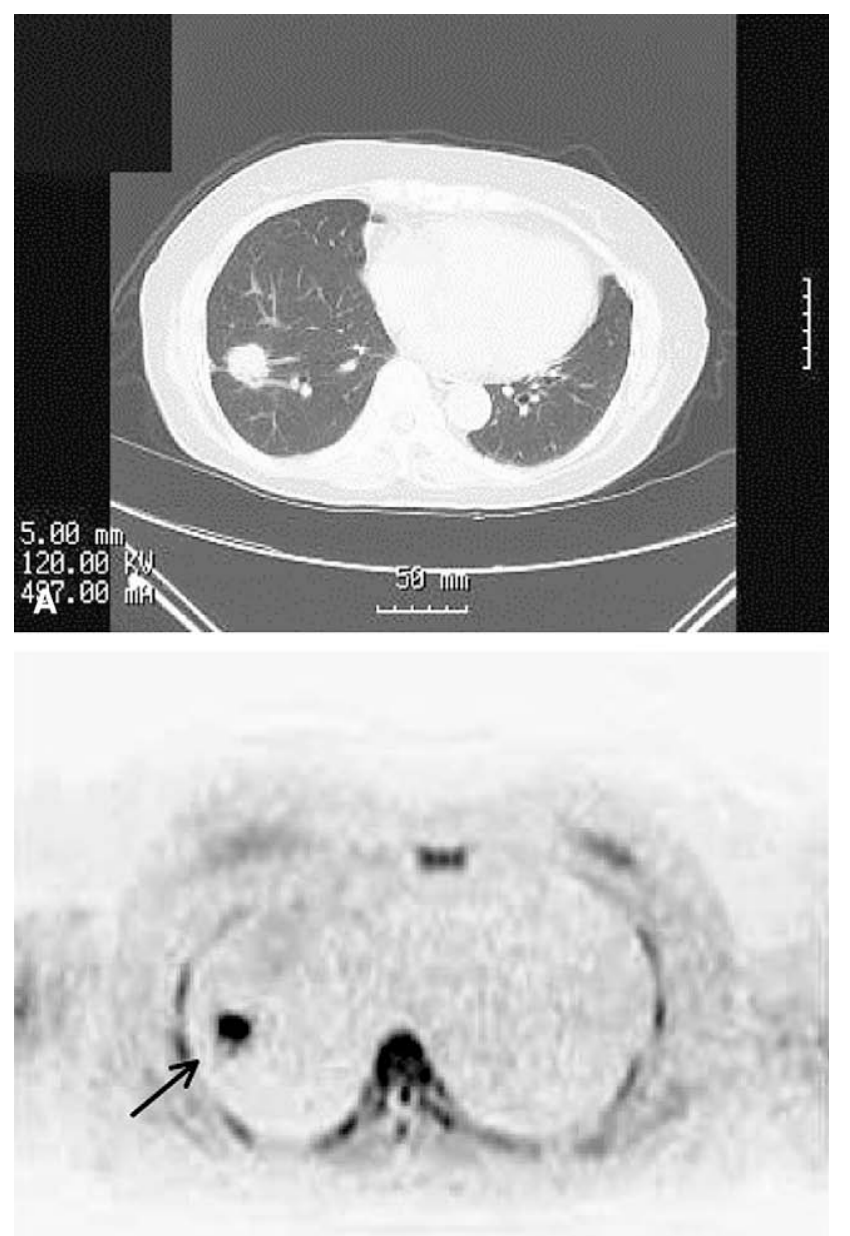

B

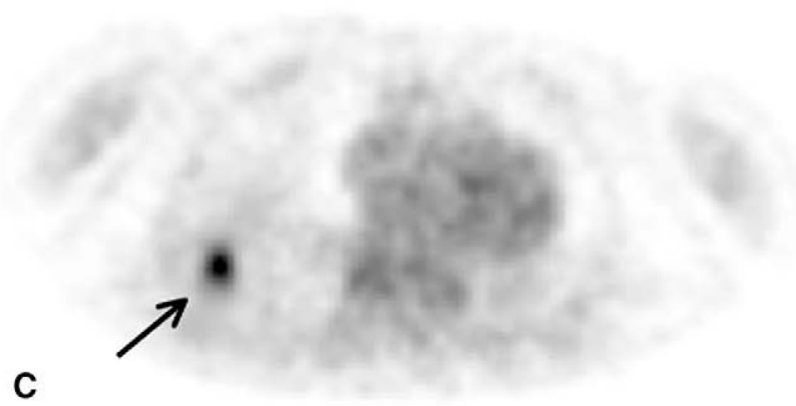

FIGURE 1. Lung adenocarcinoma in right lower lobe imaged with computed tomography (A), diffusion-weighted imaging (B), and positron emission tomography with fludeoxyglucose F 18 (C). Arrows indicate tumor images on diffusion-weighted imaging and positron emission tomography. Minimum value of apparent diffusion coefficient, maximum standard uptake value, and contrast ratio of standard uptake value in tumor are 0.98 $\times 10^{-3} \mathrm{~mm}^{2} / \mathrm{s}, 3.2$, and 0.68 , respectively.

inflammation in 20, hamartoma in 2, and other lesions in 2. There was no significant difference in mean size between NSCLC and benign lesions.

DWI clearly identified the malignant nodules in a fashion similar to PET imaging (Figure 1). The receiver operating
TABLE 2. Findings of diffusion-weighted imaging and positron emission tomography in non-small cell lung cancer

\begin{tabular}{lrrrrrr}
\hline \multirow{2}{*}{ Histologic type } & \multicolumn{2}{c}{ DWI } & & \multicolumn{2}{c}{ PET } & \\
\cline { 2 - 4 } \cline { 5 - 6 } & Positive Negative & Positive Negative & Total \\
\hline Adenocarcinoma & & & & & & \\
Well differentiated & 33 & 24 & 32 & 25 & 57 \\
Moderately differentiated & 12 & 2 & 12 & 2 & 14 \\
Poorly differentiated & 5 & 0 & 5 & 0 & 5 \\
Squamous cell carcinoma & 17 & 0 & 17 & 0 & 17 \\
Adenosquamous carcinoma & 3 & 0 & 3 & 0 & 3 \\
Total & 70 & 26 & 69 & 27 & 96 \\
\hline
\end{tabular}

DWI, Diffusion-weighted imaging; $P E T$, positron emission tomography.

characteristic curves for the benign versus malignant discrimination showed the optimal cutoff value of ADC on DWI to be $1.2 \times 10^{-3} \mathrm{~mm}^{2} / \mathrm{s}$ and the optimal cutoff value of $\mathrm{SUV}_{\mathrm{CR}}$ on PET to be 0.31 on PET.

Among the 96 NSCLC nodules, DWI results were positive for 70 and negative for 26 , whereas PET results were positive for 69 and negative for 27 (Table 2). Among the 57 well-differentiated adenocarcinomas, $24(42 \%)$ and 25 $(44 \%)$ had negative results on DWI and PET, respectively. None of the 5 poorly differentiated adenocarcinomas, 17 squamous cell carcinomas, and 3 adenosquamous carcinomas had negative results on either DWI or PET. Among the 28 benign nodules, DWI results were positive for 1 and negative for 27, whereas PET results were positive for 5 and negative for 23 (Table 3 ).

Tables 4 and 5 show the results achieved with the McNemar test. Table 4 shows the correlation between DWI and PET in diagnosis of NSCLC. There was no significant difference in sensitivity between DWI and PET $(P>.999)$. Table 5 shows the correlation between DWI and PET in diagnosis of benign nodules. There was no significant difference in specificity between DWI and PET $(P=.22)$. The accuracies of DWI and PET were 0.78 and 0.74 , respectively, with no significant difference $(P=.69)$. Positive predictive values of DWI and PET were 0.98 and 0.93 , respectively, also with no significant difference $(P=.83)$. Negative predictive values of DWI and PET were 0.51 and 0.46 , respectively, again with no significant difference $(P=.31)$.

TABLE 3. Findings of diffusion-weighted imaging and positron emission tomography in benign pulmonary nodules

\begin{tabular}{|c|c|c|c|c|c|}
\hline \multirow[b]{2}{*}{ Histologic type } & \multicolumn{2}{|c|}{ DWI } & \multicolumn{2}{|c|}{ PET } & \multirow[b]{2}{*}{ Tota } \\
\hline & Positive & Negative & Positive & Negative & \\
\hline \multicolumn{6}{|l|}{ Inflammation } \\
\hline Acute & 1 & 3 & 4 & 0 & 4 \\
\hline Chronic & 0 & 20 & 0 & 20 & 20 \\
\hline Hamartoma & 0 & 2 & 0 & 2 & 2 \\
\hline Others & 0 & 2 & 1 & 1 & 2 \\
\hline Total & 1 & 27 & 5 & 23 & 28 \\
\hline
\end{tabular}

$D W I$, Diffusion-weighted imaging; $P E T$, positron emission tomography. 
TABLE 4. Correlation between diffusion-weighted imaging and positron emission tomography for diagnosing non-small cell lung cancer

\begin{tabular}{lccc}
\hline & \multicolumn{3}{c}{ PET } \\
\cline { 2 - 4 } \multicolumn{1}{c}{ DWI } & True positive & False negative & Total \\
\hline True positive & 61 & 9 & 70 \\
False negative & 8 & 18 & 26 \\
Total & 69 & 27 & 96 \\
\hline
\end{tabular}

Sensitivity of diffusion-weighted imaging 0.73 (95\% confidence interval 0.64-0.82); sensitivity of positron emission tomography 0.72 (95\% confidence interval 0.63 $0.81) ; P>.999$ by McNemar test. $D W I$, Diffusion-weighted imaging; $P E T$, positron emission tomography.

Table 6 shows mean ADC in DWI and SUV $\mathrm{CR}_{\text {in PET }}$ with their relationships to the pathologic tumor stage, tumor invasiveness of NSCLC, and histologic differentiation in adenocarcinoma. Although DWI did not show the significant difference of the mean value of ADC in these subgroups, PET showed a significant difference in $\mathrm{SUV}_{\mathrm{CR}}$ between NSCLC at pathologic stage IA versus IB or more advanced stages; between NSCLC with and without lymphatic, vascular, and pleural involvements; and between well-differentiated and moderately or poorly differentiated adenocarcinomas $(P<.01-.001)$.

\section{DISCUSSION}

In this study, sensitivity and specificity for differentiating between NSCLC and benign pulmonary nodules were not different between DWI and PET. We therefore conclude that DWI can be used in place of PET for discrimination between benign and NSCLC lesions.

On the other hand, DWI could not predict the aggressiveness of NSCLC, including adenocarcinoma, as well as did PET. It has been reported that FDG-PET can predict the ag-
TABLE 5. Correlation between diffusion-weighted imaging and positron emission tomography for diagnosing benign pulmonary nodules

\begin{tabular}{lccr}
\hline & \multicolumn{3}{c}{ PET } \\
\cline { 2 - 4 } \multicolumn{1}{c}{ DWI } & True negative & False positive & Total \\
\hline True negative & 23 & 4 & 27 \\
False positive & 0 & 1 & 1 \\
Total & 23 & 5 & 28 \\
\hline
\end{tabular}

Specificity of diffusion-weighted imaging 0.96 (95\% confidence interval $0.90-1.03)$; specificity of positron emission tomography 0.82 ( $95 \%$ confidence interval 0.68 $0.96) ; P=.22$ by McNemar test. DWI, Diffusion-weighted imaging; $P E T$, positron emission tomography.

gressiveness of NSCLC ${ }^{15-18}$ because tumors with low aggressiveness have low rate of glucose metabolism. We previously reported that the FDG uptake could be used to predict the aggressiveness of lung adenocarcinoma; that is, lung adenocarcinomas with high FDG uptake had more frequent lymph node metastasis, higher tumor invasiveness, higher proliferative activity, and poorer prognosis than those with low FDG uptake. ${ }^{16-18}$ Because ADC in DWI of tumors is known to decrease with increased cellularity, larger nuclei, and larger nuclear/cytoplasmic ratio, ${ }^{4,5}$ we initially hypothesized that ADC might correlate with the aggressiveness of NSCLC as well as does FDG uptake in PET. In brain glioma and astrocytoma, Murakami and associates ${ }^{5}$ reported that ADC showed a significant correlation with the histologic grade of malignancy and prognosis, supporting our hypothesis. In this study, however, ADC in DWI did not show a significant correlation with pathologic tumor stage and tumor invasiveness of NSCLC or with histological differentiation of adenocarcinoma, whereas FDG uptake in PET did. We therefore conclude that although DWI can image NSCLC as well as does PET, it cannot predict the

TABLE 6. Correlation between tumor aggressiveness and minimum value of apparent diffusion coefficient and contrast ratio of standard uptake value

\begin{tabular}{|c|c|c|c|c|c|}
\hline \multirow[b]{2}{*}{ Tumor aggressiveness } & \multirow[b]{2}{*}{ No. of tumors } & \multicolumn{2}{|c|}{ DWI } & \multicolumn{2}{|c|}{ PET } \\
\hline & & ADC & $P$ value & $\mathbf{S U V}_{\mathbf{C R}}$ & $P$ value \\
\hline Tumor stage & & & .08 & & $<.001$ \\
\hline IA & 75 & $1.00 \pm 0.34$ & & $0.45 \pm 0.27$ & \\
\hline IB-IV & 21 & $0.83 \pm 0.20$ & & $0.74 \pm 0.16$ & \\
\hline Lymphatic invasion & & & .12 & & $<.01$ \\
\hline Negative & 79 & $0.98 \pm 0.34$ & & $0.46 \pm 0.27$ & \\
\hline Positive & 17 & $0.77 \pm 0.18$ & & $0.75 \pm 0.15$ & \\
\hline Vascular invasion & & & .08 & & $<.001$ \\
\hline Negative & 74 & $0.99 \pm 0.37$ & & $0.41 \pm 0.26$ & \\
\hline Positive & 22 & $0.85 \pm 0.16$ & & $0.78 \pm 0.14$ & \\
\hline Pleural involvement & & & .42 & & $<.001$ \\
\hline Negative & 80 & $0.97 \pm 0.35$ & & $0.44 \pm 0.27$ & \\
\hline Positive & 16 & $0.84 \pm 0.16$ & & $0.78 \pm 0.14$ & \\
\hline Adenocarcinoma histologic differentiation $(\mathrm{n}=76)$ & & & .11 & & $<.001$ \\
\hline Well differentiated & 57 & $1.04 \pm 0.37$ & & $0.38 \pm 0.24$ & \\
\hline Moderately or poorly differentiated & 19 & $0.89 \pm 0.22$ & & $0.61 \pm 0.25$ & \\
\hline
\end{tabular}


aggressiveness of NSCLC and of adenocarcinoma in particular.

Two reasons might explain why ADC in NSCLC did not correlate with the tumor aggressiveness, differing from the results in brain glioma and astrocytoma. ${ }^{5}$ (1) The difference in magnetic susceptibility at the tumor-lung interface creates inhomogeneity in the local magnetic field, which disrupts the MR signals of lung tumors, like the artifacts that arise from the heart-lung interface. ${ }^{26}$ (2) Respiratory and cardiac motion causes additional image artifacts and signal loss. ${ }^{27}$

One of the deficiencies of FDG-PET in diagnosis of lung cancer is its FN rate for well-differentiated adenocarcinomas, which is related to their low rate of glucose metabolism and low density. ${ }^{19}$ In this study, both DWI and PET had negative results for approximately $40 \%$ of well-differentiated adenocarcinomas, suggesting that DWI does not compensate for the PET deficiency in this regard. We therefore consider well-differentiated adenocarcinomas difficult to diagnose with either PET or DWI.

Although SUV has frequently been used for evaluation of FDG-PET, it is well known that several factors can affect the SUV, such as body size ${ }^{28}$ and blood glucose level. ${ }^{29}$ We previously compared the results of $\mathrm{SUV}_{\max }, \mathrm{SUV}_{\mathrm{CR}}$ with contralateral lung, and $\mathrm{SUV}_{\mathrm{CR}}$ with cerebellum for pulmonary nodules and reported that $\mathrm{SUV}_{\mathrm{CR}}$ with contralateral lung or cerebellum showed significantly higher sensitivity than did $\mathrm{SUV}_{\max },{ }^{20}$ a conclusion supported by Obrzut and coworkers. ${ }^{30}$ We therefore selected SUV $\mathrm{CR}_{\mathrm{CR}}$ with contralateral lung for analysis in this study.

Although FDG-PET is useful for imaging the whole body, DWI does not have that capability, because of the need for breath holding by the patient during the imaging. Recently, Takahara and colleagues ${ }^{3}$ developed a new technology of DWI that can be conducted under normal breathing conditions and allows acquisition of more slices with multiple signal averaging, higher signal-to-noise ratio, and high-quality maximumintensity projection images, ${ }^{3}$ enabling whole-body imaging. ${ }^{11}$ Ohno and colleagues ${ }^{14}$ recently reported that whole-body MRI with DWI showed similar sensitivity for M staging to PET with CT. We have also reported that DWI can be used in place of PET with CT for N staging of NSCLC. ${ }^{31}$ ADC in DWI is known to vary among MR machines, however, so as the next step a multicenter study should be conducted to determine whether whole-body DWI can be used for imaging of the systemic metastasis in lung cancer, as is FDG-PET.

This study showed that DWI can be used in place of PET for imaging NSCLC. It should be kept in mind, however, that DWI cannot predict the aggressiveness of NSCLC as well as FDG-PET does.

\section{References}

1. Wang J, Takashima S, Takayama F, Kawakami S, Saito A, Matsushita T, et al. Head and neck lesions: characterization with diffusion-weighted echo-planar MR imaging. Radiology. 2001;220:621-30.
2. Sumi M, Takagi Y, Uetani M, Morikawa M, Hayashi K, Kabasawa H, et al. Diffusion-weighted echoplanar MR imaging of salivary glands. AJR Am J Roentgenol. 2002;178:959-65.

3. Takahara T, Imai Y, Yamashita T, Yasuda S, Nasu S, Van Cauteren M. Diffusion weighted whole body imaging with background body signal suppression (DWIBS): technical improvement using free breathing, STIR and high resolution 3D display. Radiat Med. 2004;22:275-82.

4. Nasu K, Kuroki Y, Kuroki S, Murakami K, Nawano S, Moriyama N. Diffusionweighted single shot echo planar imaging of colorectal cancer using a sensitivityencoding technique. Jpn J Clin Oncol. 2004;34:620-6.

5. Murakami R, Sugahara T, Nakamura H, Hirai T, Kitajima M, Hayashida Y, et al. Malignant supratentorial astrocytoma treated with postoperative radiation therapy: prognostic value of pretreatment quantitative diffusion-weighted MR imaging. Radiology. 2007;243:493-9.

6. Woodhams R, Matsunaga K, Kan S, Hata H, Ozaki M, Iwabuchi K, et al. ADC mapping of benign and malignant breast tumors. Magn Reson Med Sci. 2005;4:35-42.

7. Hayashida Y, Yakushiji T, Awai K, Katahira K, Nakayama Y, Shimomura O, et al. Monitoring therapeutic responses of primary bone tumors by diffusionweighted image: initial results. Eur Radiol. 2006;16:2637-43.

8. Reinsberg SA, Payne GS, Riches SF, Ashley S, Brewster JM, Morgan VA, et al. Combined use of diffusion-weighted MRI and 1H MR spectroscopy to increase accuracy in prostate cancer detection. AJR Am J Roentgenol. 2007; 188:91-8.

9. Dzik-Jurasz A, Domenig C, George M, Wolber J, Padhani A, Brown G, et al. Diffusion MRI for prediction of response of rectal cancer to chemoradiation. Lancet. 2002;360:307-8.

10. Matoba M, Tonami H, Kondou T, Yokota H, Higashi K, Toga H, et al. Lung carcinoma: diffusion weighted MR imaging-preliminary evaluation with apparent diffusion coefficient. Radiology. 2007;243:570-7.

11. Komori T, Narabayashi I, Matsumura K, Matsuki M, Akagi H, Ogura Y, et al. 2[Fluorine-18]-fluoro-2-deoxy-D-glucose positron emission tomography/computed tomography versus whole-body diffusion-weighted MRI for detection of malignant lesions: initial experience. Ann Nucl Med. 2007;21:209-15.

12. Lichy MP, Aschoff P, Plathow C, Stemmer A, Horger W, Mueller-Horvat C, et al. Tumor detection by diffusion-weighted MRI and ADC-mapping. Initial clinical experiences in comparison to PET-CT. Invest Radiol. 2007;42:605-13.

13. Mori T, Nomori H, Ikeda K, Kawanaka K, Shiraishi S, Katahira K, et al. Diffusion-weighted magnetic resonance imaging for diagnosing malignant pulmonary nodules/masses. J Thorac Oncol. 2008;3:358-64.

14. Ohno Y, Koyama H, Onishi Y, et al. Non-small cell lung cancer: wholebody MR examination for M-stage assessment-utility for whole-body diffusion-weighted imaging compared with integrated FDG PET/CT. Radiology. 2008;248:643-54

15. Cerfolio RJ, Bryant AS, Ohja B, Bartolucci AA. The maximum standardized uptake values on positron emission tomography of a non-small cell lung cancer predict stage, recurrence, and survival. J Thorac Cardiovasc Surg. 2005; 130:151-9.

16. Nomori H, Watanabe K, Ohtsuka T, Naruke T, Suemasu K, Kobayashi T, et al. Fluorine 18-tagged fluorodeoxyglucose positron emission tomographic scanning to predict lymph node metastasis, invasiveness, or both, in clinical T1 N0 M0 lung adenocarcinoma. J Thorac Cardiovasc Surg. 2004;128:396-401.

17. Ohtsuka T, Nomori H, Watanabe K, Kaji M, Naruke T, Suemasu K, et al. Prognostic significance of $[18 \mathrm{~F}]$ fluorodeoxyglucose uptake on positron emission tomography in patients with pathologic stage I lung adenocarcinoma. Cancer. 2006; 107:2468-73.

18. Watanabe K, Nomori H, Ohtsuka T, Naruke T, Ebihara A, Orikasa H, et al. [F-18] Fluorodeoxyglucose positron emission tomography can predict pathological tumor stage and proliferative activity determined by $\mathrm{Ki}-67$ in clinical stage IA lung adenocarcinomas. Jpn J Clin Oncol. 2006;36:403-9.

19. Menda Y, Bushnell DL, Madsen MT, McLaughlin K, Kahn D, Kernstine KH. Evaluation of various corrections to the standardized uptake value for diagnosis of pulmonary malignancy. Nucl Med Commun. 2001;22:1077-81.

20. Nomori H, Watanabe K, Ohtsuka T, Naruke T, Suemasu K, Uno K. Evaluation of F-18 fluorodeoxyglucose (FDG) PET scanning for pulmonary nodules less than $3 \mathrm{~cm}$ in diameter, with special reference to CT images. Lung Cancer. 2004;45: 19-27.

21. Nomori H, Watanabe K, Ohtsuka T, Naruke T, Suemasu K, Uno K. Visual and semiquantitative analyses for F-18 fluorodeoxyglucose PET scanning in pulmonary nodules $1 \mathrm{~cm}$ to $3 \mathrm{~cm}$ in size. Ann Thorac Surg. 2005;79:984-8.

22. Koh DM, Padhani AR. Diffusion-weighted MRI: a new functional clinical technique for tumor imaging. Br J Radiol. 2006;79:633-5. 
23. Sobin LW, Witteking CH. UICC TMN classification of malignant tumors. 6th ed. New York: Wiley-Liss; 2002. p. 131-41.

24. Bréchot JM, Chevret S, Charpentier MC, Appere de Vecchi C, Capron F, Prudent J, et al. Blood vessel and lymphatic vessel invasion in resected non-small cell lung carcinoma. Cancer. 1996;78:2111-8.

25. Takise A, Kodama T, Shimosato Y, Watanabe S, Suemasu K. Histopathologic prognostic factors in adenocarcinomas of the peripheral lung less than $2 \mathrm{~cm}$ in diameter. Cancer. 1988;61:2083-8.

26. Atalay MK, Poncelet BP, Kantor HL, Brady TJ, Weisskoff RM. Cardiac susceptibility artifacts arising from the heart-lung interface. Magn Reson Med. 2001;45:341-5.

27. Chen BT, Yordanov AT, Johnson GA. Ventilation-synchronous magnetic resonance microscopy of pulmonary structure and ventilation in mice. Magn Reson Med. 2005;53:69-75.
28. Kim CK, Gupta NC, Chandramouli B, Alavi A. Standardized uptake values of FDG: body surface area correction is preferable to body weight correction J Nucl Med. 1994;35:164-7.

29. Lindholm P, Minn H, Leskinen-Kallio S, Bergman J, Ruotsalainen U, Joensuu H Influence of the blood glucose concentration on FDG uptake in cancer-a PET study. J Nucl Med. 1993;34:1-6.

30. Obrzut S, Pham RH, Vera DR, Badran K, Hoha CK. Comparison of lesion-to-cerebellum uptake ratios and standardized uptake values in the evaluation of lung nodules with 18F-FDG PET. Nucl Med. 2007;27:7-13.

31. Nomori H, Mori T, Ikeda K, Kawanaka K, Shiraishi S, Katahira K, et al Diffusion-weighted magnetic resonance imaging can be used in place of positron emission tomography for $\mathrm{N}$ staging of non-small cell lung cancer with fewer false-positive results. J Thorac Cardiovasc Surg. 2008;135:816-22. 